\title{
The Lagrangian Formulation and Gauge Theory of the Standard Model
}

\author{
Mohammed Ibrahim Abdelgabar ${ }^{1 *}$, Abdulhamid Yakubu², Mubarak Dirar Abdallah ${ }^{3}$ \\ ${ }^{1}$ Sudan University of Science and Technology, Khartoum State, Sudan \\ ${ }^{2}$ Department of Physics International University of Africa, Khartoum, Sudan \\ ${ }^{3}$ Dpatrment of Physics, Sudan University of Sciences and Technology, Khartoum State, Sudan \\ Email: *moha1175@yahoo.com
}

How to cite this paper: Abdelgabar, M.I., Yakubu, A. and Abdallah, M.D. (2021) The Lagrangian Formulation and Gauge Theory of the Standard Model. Open Access Library Journal, 8: e7875.

https://doi.org/10.4236/oalib.1107875

Received: August 20, 2021

Accepted: September 27, 2021

Published: September 30, 2021

Copyright $\odot 2021$ by author(s) and Open Access Library Inc.

This work is licensed under the Creative Commons Attribution International License (CC BY 4.0).

http://creativecommons.org/licenses/by/4.0/

\section{(c) (i) Open Access}

\begin{abstract}
Study of gauge symmetry is carried over the different interacting and non-interacting field theoretical models through a prescription based on Lagrangian formulation. And the prescription is capable of testing whether a given model possesses a gauge symmetry or not. It can successfully formulate the gauge transformation generator in all the cases whatever subtleties are involved in it. It is found that the prescription has the ability to show a direction how to extend the phase space using auxiliary fields to restore the gauge invariance of a theory. Like the usual phase space, the prescription is found to be equally powerful in the extended phase space of a theory.
\end{abstract}

\section{Subject Areas}

Applied Physics

\section{Keywords}

Symmetry, Standard Model, Lagrangian, Particle Physics, Elementary Particles

\section{Introduction}

The most important model in particle physics now is the standard model; it describes the weak, strong and electromagnetic interactions between quarks and leptons which are now said to be the elementary particles of atom.

Particle physics is the science of the smallest constituents of matter, and how they interact. It evolves from physical chemistry, where it was first resolving that all chemical substances, like molecules, are made up of chemical elements, At the turn of $19^{\text {th }}$ to $20^{\text {th }}$ century, it was then found out that the atoms were not elementary, rather they had constituents, a single nucleus and a number of indis- 
tinguishable electrons [1].

Early $20^{\text {th }}$ century, it was then found that nucleus was not elementary but a constitution of proton and neutron. Later on, constitutes of neutron and proton were revealed to be quarks. Consequently, that gave rise of the standard model that answered the unresolved questions, by mathematical formulations and theories. One of the most crucial formulations is the Lagrangian that describes the behaviour of a system, with the concept of relativity and quantum mechanics [2].

And the main objective of this manuscript is a description of the interaction between fundamental particles in terms of the exchange of fundamental force particles and gauge coupling on the view of standard model (SM).

\section{Elementary Particles}

Elementary particles are then classified into two according to energy state statistics. Elementary particles that obey Bose-Einstein statistics are called Bosons i.e. there can be arbitrary many of them in any given state of fixed quantum numbers, they also have spin $(n \hbar)$. While Fermions are those that obey Fermi-Dirac statistics and have to adhere to the Pauli principle i.e. only single fermion can be in any given state with fixed quantum number, and they have spin $\left(\frac{n+1}{2}\right) \hbar$ [3].

Elementary particles are known to be 38 in number; 24 are Fermions, 13 Bosons and 1 Higgs. The union of special relativity and quantum mechanics that describes these particles is called quantum field theory [4].

Standard model explains that fermions are the constituents of matter which are subdivided into two: Quarks and Leptons and gauge bosons mediate their interactions. Quarks are six particles same as Leptons with anti-particles of the same properties except for charge. These twelve particles are of three families or generations naturally.

1) Electron neutrino $\left(v_{e}\right)$, electron $\left(e^{-}\right)$, up quark $(u)$, down quark $(d)$;

2) Muon neutrino $\left(v_{\mu}\right)$, muon $\left(\mu^{-}\right)$, charm Quark $(c)$, strange quark $(s)$;

3) Tau neutrino $\left(v_{\tau}\right)$, Tau $(\tau)$, top quark $(t)$, bottom quark $(b)$.

Each generation consists of two leptons with charges $Q=0$ and $Q=-1$ and two quarks with charges $Q=+\frac{2}{3}$ and $Q=-\frac{1}{3}$.

All visible matter in the universe is made from the first generation of matter particles, up quarks, down quarks, and electrons. This is because all second and third generation particles are unstable and quickly decay into stable first-generation particles [5].

1) Colour charge: a question may rise, why one saw only those combinations of quarks and antiquarks that had integer charge, and why no one ever saw a $\mathrm{q}$, q-q, q-q-q-bar, or countless other combinations [6].

\section{Range of the Forces}

The range or limitation of a force explains the distance covered by that force and 
where you cannot find that force. The range of forces is related to the mass of exchange particle $\mathrm{M}$ [7].

An amount of energy $\Delta E=m c^{2}$ borrowed for a time $\Delta t$ is governed by the Uncertainty Principle:

$$
\Delta E \cdot \Delta T \sim \hbar
$$

The maximum distance the particle can travel is $\Delta \chi=c \Delta t$, where $c$ is velocity of light.

$$
\begin{gathered}
\Delta \chi=\hbar c / \Delta E \\
\Delta \chi=\hbar c / M c^{2} \text { forces' range formula. }
\end{gathered}
$$

The photon has $M=0$, which shows infinite range of EM force.

W boson has a mass of $80 \mathrm{GeV} / \mathrm{c}^{2}$, Range of weak force is

$$
197 \mathrm{MeV} \mathrm{fm} / 8 \times 10^{5} \mathrm{MeV}=2 \times 10^{-3} \mathrm{fm}[8] .
$$

\subsection{Gauge Theory (Symmetry)}

A gauge theory is one that possesses invariance under a set of local transformations", i.e. transformations whose parameters are space-time dependent [9].

In the case of quantum electrodynamic (QED), the gauge symmetry is abelian, [10] in this case the gauge transformations are local complex phase transformations of the fields of charged particles, and gauge invariance necessitates the introduction of a massless vector (spin-1) particle, called the photon, whose exchange mediates the electromagnetic interactions [10].

In the 1950's Yang and Mills considered (as a purely mathematical exercise) extending gauge invariance to include local non-abelian (i.e. non-commuting) transformations such as SU (2).

In this case one needs a set of massless vector fields (three in the case of SU (2)), which were formally called "Yang-Mills" fields, but are now known as "gauge fields".

\subsection{Gauge Transformation for QED}

Consider the Lagrangian density for a free Dirac field $\psi$.

$$
\mathcal{L}=\bar{\Psi}\left(i \gamma^{\mu} \partial_{\mu}-m\right) \Psi
$$

This Lagrangian density is invariant under a phase transformation of the fermion field

$$
\begin{gathered}
\Psi \rightarrow \mathrm{e}^{i Q \omega} \Psi, \bar{\Psi}=\mathrm{e}^{i Q \omega} \bar{\Psi}, \\
Q \Psi=+\Psi, \quad Q \bar{\Psi}=-\bar{\Psi}
\end{gathered}
$$

where $Q$ is the charge operator $\omega$ is a real constant (i.e. independent of $x$ ) and $\bar{\psi}$ is the conjugate field.

The set of all numbers $\mathrm{e}^{-i \omega}$ form a group2. This particular group is "abelian" which is tosay that any two elements of the group commute. This just means that [11] 


$$
\mathrm{e}^{-i \omega_{1}} \mathrm{e}^{-i \omega_{2}}=\mathrm{e}^{-i \omega_{2}} \mathrm{e}^{-i \omega_{1}}
$$

This particular group is called $\mathrm{U}(1)$ which means the group of all unitary $1 \times 1$ matrices. A unitary matrix satisfies $U^{\uparrow}=U^{-1}$ with $U^{\uparrow}$ being the adjoint matrix.

We can now state the invariance of the Lagrangian Equation (1.1) under phase transformations in amore fancy way by saying that the Lagrangian is invariant under global U (1) transformations. By global we mean that $\omega$ does not depend on $X$.

We assume that the parameter $\omega$ is sufficiently small that we can expand in $\omega$ and neglect all but the linear term. Thus, we write

$$
\mathrm{e}^{-i \omega}=1-i \omega+O\left(\omega^{2}\right)
$$

Under such infinitesimal phase transformations, the field $\psi$ changes according to:

$$
\Psi \rightarrow \Psi+\delta \Psi=\Psi+i Q \omega \Psi
$$

and the conjugate field $\bar{\psi}$ by

$$
\bar{\Psi} \rightarrow \bar{\Psi}+\delta \bar{\Psi}=\bar{\Psi}+i Q \omega \bar{\Psi}=\bar{\Psi}-i \omega \bar{\Psi}
$$

such that the Lagrangian density remains unchanged (to order $\omega$ ).

At this point we should note that global transformations are not very attractive from a theoretical point of view. The reason is that making the same transformation at every space-time point requires that all these points 'know' about the transformation [12].

Thus, we have two options at this point. Either, we simply note the invariance of Equation (1.1) under global U (1) transformations and put this aside as a curiosity, or we insist that invariance under gauge transformations is a fundamental property of nature. If we take the latter option, we have to require invariance under local transformations. Local means that the parameter of the transformation, $\omega$, now depends on the space-time point $x$. Such local (i.e. space-time dependent) transformations are called "gauge transformations".

If the parameter $\omega$ depends on the space-time point then the field $\psi$ transforms as follows under infinitesimal transformations

$$
\delta \Psi(x)=i \omega(x) \Psi(x) ; \delta \bar{\Psi}=-i \omega(x) \bar{\Psi}(x) .
$$

Note that the Lagrangian density Equation (1.1) now is no longer invariant under these transformations, because of the partial derivative between $\bar{\psi}$ and $\psi$. This derivative will act on the space-time dependent parameter $\omega(x)$ such that the Lagrangian density changes by an amount $\delta \mathcal{L}$, where

$$
\delta \mathcal{L}=-\bar{\Psi}(x) \gamma^{\mu}\left(\partial_{\mu} Q \omega(x)\right) \Psi(x) .
$$

The square brackets in $\left[\partial_{\mu} Q_{\omega}(x)\right]$ are introduced to indicate that the derivative $\partial_{\mu}$ acts only inside the brackets. It turns out that we can restore gauge invariance if we assume that the fermion field interacts with a vector field $A_{\mu}$, called a "gauge field", with an interaction term [13] 


$$
-e \bar{\psi} \gamma^{\mu} A_{\mu} Q \psi
$$

Added to the Lagrangian density which now becomes:

$$
\mathcal{L}=\bar{\psi}\left(i \gamma^{\mu}\left(\partial_{\mu}+i e Q A_{\mu}\right)-m\right) \psi
$$

In order for this to work we must also assume that apart from the fermion field transforming under a gauge transformation according to Equation (1.7) the gauge field, $A_{\mu}$, also changes according to

$$
-e Q A_{\mu} \rightarrow-e Q\left(A_{\mu}+\delta A_{\mu}(x)\right)=-e Q A_{\mu}+Q \partial_{\mu} \omega(x)
$$

So $\delta A_{\mu}(x)=\frac{-Q \partial_{\mu} \omega(x)}{e}$.

This change exactly cancels with Equation (1.8), so that once this interaction term has been added the gauge invariance is restored. We recognize Equation (1.10) as being the fermionic part of the Lagrangian density for QED, where $e$ is the electric charge of the fermion and $A_{\mu}$ is the photon field [14].

In order to have a proper quantum field theory, in which we can expand the photon field $A_{\mu}$ in terms of creation and annihilation operators for photons, we need a kinetic term for the photon, i.e. a term which is quadratic in the derivative of the field $A_{\mu}$. Without such a term the Euler-Lagrange equation for the gauge field would be an algebraic equation and we could use it to eliminate the gauge field altogether from the Lagrangian. We need to ensure that in introducing a kinetic term we do not spoil the invariance under gauge transformations.

This is achieved by defining the field strength tensor, $F_{\mu \text { r }}$ as

$$
F_{\mu v}=\partial_{\mu} A_{v}-\partial_{v} A_{\mu}
$$

where the derivative is understood to act on the A-field only. It is easy to see that under the gauge transformation Equation (1.11) each of the two terms on the right-hand side of Equation (1.12) change, but the changes cancel out. Thus, we may add to the Lagrangian any term which depends on $F_{\mu \nu}$ (and which is Lorentz invariant, thus, with all Lorentz indices contracted) [15].

Such a term is a $F_{\mu v} F_{\mu v}$. This gives the desired term which is quadratic in the derivative of the field $A_{\mu}$. If we choose the constant a to be $-1 / 4$ then the Lagrange equations of motion match exactly (the relativistic formulation of) Maxwell's equations.

We have thus arrived at the Lagrangian density for QED, but from the viewpoint of demanding invariance under $U$ (1) gauge transformations rather than starting with Maxwell's equations and formulating the equivalent quantum field theory.

The Lagrangian density for QED is:

$$
\mathcal{L}=-\frac{1}{4} F_{\mu \nu} F^{\mu \nu}+\bar{\psi}\left(i \gamma^{\mu}\left(\partial_{\mu}+i e Q A_{\mu}\right)-m\right) \psi
$$

Or we can say the Lagrangian for a fermion coupled to a $U$ (1) gauge field is quantum electrodynamics (QED), if we call the fields electron and photon: [16] 


$$
\mathcal{L}_{\mathrm{QED}}=\frac{1}{4} F_{\mu \nu} F^{\mu v}+\bar{\psi}(i \mathcal{D}-m)
$$

where

$$
i D=\left(i \gamma^{\mu}\left(\partial_{\mu}+i e Q A_{\mu}\right)\right)
$$

\subsection{Gauge Transformation for QCD}

QCD is the theory of the strong interaction, [17] strong interaction is mediated by gluons, they are massless, spin-1 particles with two polarization states (left-handed and right-handed). They are represented by a four-component vector potential $A_{\mu}(x)$ with a Lorentz index $\mu=0,1,2,3$, just as in electromagnetism. Therefore, conditions must be imposed on $A_{\mu}(x)$ to select only the physical degrees of freedom, as different $A_{\mu}(x)$ can give rise to the same physics. These conditions are called gauge conditions or gauge choices. For the same reason, $A_{\mu}(x)$ are called gauge potentials, and gluons are called gauge particles.

QCD is an unbroken gauge theory, belonging to the group of non-abelian SU (3) [18]. There are 8 types of gluons mediating the strong interactions. This proliferation of gauge particles has to do with the SU (3) colour symmetry.

It is easy to see that L is invariant under a global SU (3) transformation

$$
\psi^{\prime}(x)=U \psi(x)
$$

where $U$ is a $3 \times 3$ unitary matrix acting on colour index, and "global" means that the field at different spacetime is transformed in exactly the same way. A generic SU (3) matrix requires 8 real parameters, usually written in the form [19]

$$
U=\exp \left(i \sum_{a} \theta^{a} \lambda^{a} / 2\right)
$$

where $\lambda^{a} / 2(a=1, \cdots, 8)$ are $3 \times 3$ hermitian matrices and are called generators of SU (3) rotations.

If we introduce 8 gluon potentials, $A_{\mu}$ a, as well as the associated covariant derivative, $D_{\mu} \equiv \partial_{\mu}+i g A_{a}^{\mu} \lambda^{a} / 2$, the free quark lagrangian can be modified to

$$
\mathcal{L}_{q}=\bar{\psi}\left(i \mathcal{D}-m_{q}\right) \psi
$$

According to Maxwell theory on magnetism anti symmetric field strength tensor $F_{\mu v a}$ and the kinetic energy term for the gluons are

$$
\mathcal{L}_{g}=\frac{1}{4} F_{\mu v a} F^{\mu v a}
$$

The above equation is the Lagrangian for the gluon.

The full QCD Lagrangian density is the sum of quark and gluon term

$$
\begin{gathered}
\mathcal{L}_{\mathrm{QCD}}=\mathcal{L}_{q}+\mathcal{L}_{g} \\
\mathcal{L}_{\mathrm{QCD}}=\bar{\psi}\left(i \mathcal{D}-m_{q}\right) \psi-\frac{1}{4} F^{\mu v a} F_{\mu v a}
\end{gathered}
$$

\subsection{Gauge Transformation for Weak Interaction}

Gauge transformation of weak interaction is similar to QCD, only which differs 
from it as discussed in chapter two that it is of $2 \times 2$ matrix and it is mediated by 3 -gauge bosons [20].

$$
\mathcal{L}_{\text {WEAK }}=\bar{\psi}\left(i \gamma^{\mu} \partial_{\mu}-m\right) \psi-g\left(\bar{\psi} \gamma^{\mu} \frac{\tau_{\alpha}}{2} \psi\right) W_{\mu}^{\alpha}-\frac{1}{4} W_{\mu \nu}^{\alpha} W_{\alpha}^{\mu \nu}
$$

\section{Conclusions}

It is discussed in this paper, what is meant by standard model, how it comes to existence and also the most crucial part of it.

Clear explanation is made right from the history of it, to the emergence of elementary particles, and what are known to be elementary now and then. A wide explanation was made about the theories that explained the four fundamental forces, followed by explaining that they are part of gauge theory, and at last deriving their Lagrangian formulation.

This paper can serve as an introduction to the model as well as a good productive to someone well acquainted to the model.

Finally my grateful thanks to my teachers and my colleagues in the International University of Africa (IUA)-Physics Department and my the staff of physics in the Sudan University of Science and Technology (SUST)for their great help.

\section{Conflicts of Interest}

The authors declare no conflicts of interest.

\section{References}

[1] Axel Maas (2013) Introduction to Particle Physics Lecture at the FSU Jena.

[2] Hollik June, W. (2009) Quantum Field Theory and the Standard Model.

[3] Malham, S.J.A. (2016) An Introduction to Lagrangian and Hamiltonian Mechanics.

[4] Martin, B.R. (2006) Nuclear and Particle Physics. John Wiley \& Sons, Ltd., London.

[5] Fuks, B. (2011) Standard Model of Particle Physics and beyond IPHC Strasbourg. University of Strasbourg, Strasbourg.

[6] Emmanuel (2015) Introduction to Particle Physics. Physics Dept University of Athens CERN.

[7] Greenwood, N.D.A. (2007) An Introduction to the Standard Model of Particle Physics. Cambridge University Press, New York. https://doi.org/10.1017/CBO9780511791406

[8] Schellekens, A.N. (2017) Last Modified beyond the Standard Model. https://www.nikhef.nl/en/activities/phd/

[9] Teubner, T. (2014) The Standard Model. Department of Mathematical Sciences University of Liverpool, Liverpool.

[10] Barr, A.J. (2014) The Standard Model.

[11] Bettoni, D. (2011) The Standard Model Lagrangian Elementary Particle Physics Strong Interaction Fenomenology. National Institute of Nuclear Physics, Legnaro.

[12] Schwartz, M.D. (2014) Quantum Field Theory and the Standard Model. Cambridge 
University Press, Cambridge.

[13] Acharya, B.S. (2016) The Standard Model of Particle Physics. International Centre for Theoretical Physics and King's College London African School of Kigali, Kigali.

[14] Crespo-Anadón, J.I. (2017) Particle Physics: History of Particle Physics Columbia University Science Honors Program.

[15] Buchmuller, W. and Ludeling, C. (2015) Field Theory and Standard Model. Deutsches Elektronen-Synchrotron DESY, Hamburg.

[16] Seymour, M.H. (1996) Particle Physics by Eugene Kenned Quantum Chromo-Dynamics. School of Physics and Astronomy, University of Manchester, Manchester.

[17] Williams, A.G. (2003) Introduction QCD and the Standard Model. Progress of Theoretical Physics Supplement, 151, 21-31. https://doi.org/10.1143/PTPS.151.21

[18] Uwe-Jens (2018) The Standard Model of Particle Physics. Theoretical Physics University of Bern, Bern.

[19] Wong, S.S.M. (2004) Introductory Nuclear Physics. Second Edition, Wiley-VCH, Hoboken.

[20] Abdalgabar, A.I. (2017) Overview on the Standard Model of Particle Physics. Department of Physics, Sudan University of Science and Technology, Khartoum. 